\title{
Tarifpolitischer Jahresbericht 2008: Tarifpolitik in der Finanzmarktkrise
}

Das Tarifjahr 2008 zeigt im Rückblick ein gespaltenes Bild: Im ersten Halbjahr standen die Tarifauseinandersetzungen unter dem Eindruck der noch guten Konjunktur. Die lohnpolitische Erwartungshaltung der Beschäftigten war angesichts der vielfach positiven Gewinnsituation entsprechend hoch. In einer Reihe von Branchen gelang es den Gewerkschaften, deutlich höhere Tarifabschlüsse als in den Vorjahren zu tätigen. In der zweiten Jahreshälfte gewann die Finanzmarktkrise an Bedeutung, vor allem für die Tarifrunde in der Metall- und Elektroindustrie. Insgesamt präsentierten sich die Gewerkschaften in zahlreichen (Warn-)Streikaktionen mit hoher Beteiligung kampfbereit. Die umstrittene Regulierung des Niedriglohnsektors kam nur wenig voran.

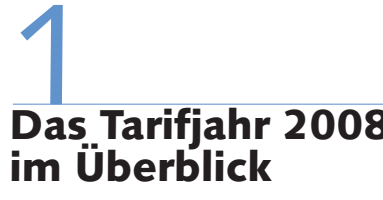

\subsection{FORDERUNGEN}

Für die Tarifrunde 2008 entscheidend waren die anhaltend positive Gewinnentwicklung der privaten Wirtschaft, die kräftige, teils exzessive Steigerung der Manager- und Vorstandsbezüge und das bei den Beschäftigten ausgeprägte Gefühl, am jahrelangen Aufschwung bislang nicht oder nur unzureichend partizipiert zu haben. Die daraus resultierende Erwartungshaltung der Beschäftigten fand ihren Niederschlag auch in den Tarifforderungen. Sie wurden zusätzlich durch die stark steigenden Verbraucherpreise getrieben, die im Laufe des ersten Halbjahres eine Zuwachsrate von bis zu 3,3 \% im Vorjahresvergleich erreichten. Die Lohn- und Gehaltsforderungen der Gewerkschaften fielen deshalb 2008 noch einmal höher aus als im Vorjahr. Spitzenreiter war der öffentliche Dienst. Dort forderte die Vereinte Dienstleistungsgewerkschaft (ver.di) eine Tarifanhebung von $8 \%$, mindestens jedoch $200 €$. Ebenfalls $8 \%$ forderten die Gewerkschaften unter anderem in der Stahlindustrie, in der Metallindustrie, im Bankgewerbe und in der Papierverarbeitung. Erstmals seit 2004 stellte die IG BCE für die chemische Industrie mit $7 \%$ wieder eine bezifferte Forderung auf. In anderen Branchen blieben die Forderungen mit

\begin{tabular}{lc}
\hline Übersicht 1: Tarifforderungen in der Tarifrunde 2008 in ausgewählten \\
Tarifbereichen - in \% - \\
\hline Bankgewerbe & 8,0 \\
& $($ mind. 260 €) \\
Chemische Industrie & 7,0 \\
Deutsche Post AG & 7,0 \\
Eisen- und Stahlindustrie & 8,0 \\
Kfz-Gewerbe Nordrhein-Westfalen & 5,0 \\
Landwirtschaft & 5,5 \\
Metallindustrie & 8,0 \\
Nahrung-Genuss-Gaststätten & $4,5-6,0$ \\
Öffentlicher Dienst (Bund, Gemeinden) & 8,0 \\
& $($ mind. 200 $€$ ) \\
Papier verarbeitende Industrie & 8,0 \\
Redakteure an Tageszeitungen und Zeitschriften & 7,5 \\
Textil- und Bekleidungsindustrie & 5,5 \\
\hline & \\
Quelle: WsI-Tarifarchiv. & WSI MITTEILUNGEN \\
\hline
\end{tabular}

4,5\% bis 5,5\% deutlich darunter (Übersicht 1).

Nach dem Kündigungsterminkalender liefen die Tarifverträge im öffentlichen Dienst (Bund, Gemeinden) Ende Dezember 2007 als erste aus, gefolgt von der Eisenund Stahlindustrie Ende Januar 2008. Die Entgelttarifverträge in der chemischen Industrie liefen regional unterschiedlich zwischen Ende Februar und Ende April aus. Die Verträge im westdeutschen Kfz-Gewerbe endeten überwiegend ebenfalls Ende Februar.

\subsection{ABSCHLÜSSE}

Den ersten wichtigen Abschluss gab es am 20.2.2008 nach intensiven Warnstreiks in der Eisen- und Stahlindustrie. Für Februar wurde eine Pauschale von $200 €$ gezahlt, zum 1.3.2008 folgte eine Tarifanhebung um 5,2 \% mit einer Laufzeit bis Ende März 2009. Am 31.3.2008 vereinbarten die Tarifparteien im öffentlichen Dienst (Bund, Gemeinden) einen Sockelbetrag von $50 €$ sowie eine Anhebung um 3,1\% ab dem 1.1.2008 (für die Gemeinden Ost erfolgte beides ab 1.4.2008), es ging weiter mit einer Einmalzahlung von $225 €$ zum Jahresbeginn 2009 und einer Stufenerhöhung von

Reinhard Bispinck, Dr., Wissenschaftler im
WSI und Leiter des WSI-Tarifarchivs in der
Hans-Böckler-Stiftung.
e-mail: Reinhard-Bispinck@boeckler.de
Götz Bauer, Monika Müller, Ulrich Schmidt,
Monika Schwacke-Pilger, Andrea Taube und
Monika Wiebel sind Sachbearbeiterinnen
und Sachbearbeiter im WSI-Tarifarchiv.




\begin{tabular}{|c|c|c|}
\hline \multicolumn{3}{|c|}{$\begin{array}{l}\text { Übersicht 2: Ausgewählte Lohn- und Gehaltsabschlüsse West und Ost } \\
\text { für } 2008\end{array}$} \\
\hline Abschluss & Tarifbereich & Ergebnis \\
\hline 23.01 .2008 & Landwirtschaft & $\begin{array}{l}\text { Bundesempfehlung: } \\
\text { 3,8 \% ab 01.03.08 } \\
\text { 3,3\% Stufenerhöhung ab 01.02.09, Laufzeit bis 31.03.10 }\end{array}$ \\
\hline 20.02 .2008 & $\begin{array}{l}\text { Eisen- und Stahlindustrie } \\
\text { Niedersachsen, Bremen, } \\
\text { Nordrhein-Westfalen }\end{array}$ & $\begin{array}{l}200 € \text { Pauschale für Februar } 2008 \\
5,2 \% \text { ab 01.03.08, Laufzeit bis 31.03.09 }\end{array}$ \\
\hline 21.02 .2008 & $\begin{array}{l}\text { Hotel- und Gaststätten- } \\
\text { gewerbe Nordrhein- } \\
\text { Westfalen }\end{array}$ & $\begin{array}{l}3,0 \% \text { ab 01.03.08 } \\
\mathbf{2 , 5} \% \text { Stufenerhöhung ab 01.03.09, Laufzeit bis 31.05.10 }\end{array}$ \\
\hline 07.03.2008 & Kfz-Gewerbe Hessen & $\begin{array}{l}\text { Nach einem Nullmonat (März) } \\
\mathbf{2 , 5} \% \text { ab 01.04.08 } \\
\mathbf{2 , 0} \% \text { Stufenerhöhung ab 01.12.08 } \\
\mathbf{2 , 0} \% \text { Stufenerhöhung ab 01.12.09, Laufzeit bis } 30.04 .10\end{array}$ \\
\hline 11.03 .2008 & $\begin{array}{l}\text { Textil- und Bekleidungs- } \\
\text { industrie West }\end{array}$ & $\begin{array}{l}\text { Nach einem Nullmonat (März) } \\
200 € \text { Pauschale insgesamt für April und Mai } 2008 \\
3,6 \% \text { ab 01.06.08, Laufzeit bis 28.02.09 }\end{array}$ \\
\hline 31.03 .2008 & $\begin{array}{l}\text { Öffentlicher Dienst } \\
\text { Bund, Gemeinden } \\
\text { West und Ost }\end{array}$ & $\begin{array}{l}50 € \text { Sockelbetrag plus } \\
3,1 \% \text { ab 01.01.08 (Gemeinden Ost: beides ab 01.04.08) } \\
2,8 \% \text { Stufenerhöhung ab 01.01.09 } \\
225 € \text { Einmalzahlung zum 01.01.2009, Laufzeit bis } 31.12 .09 \\
\text { (Abweichungen bei Krankenhäusern, Nahverkehr und } \\
\text { Versorgung) }\end{array}$ \\
\hline 16.04 .2008 & $\begin{array}{l}\text { Chemische Industrie } \\
\text { West }\end{array}$ & $\begin{array}{l}4,4 \% \text { regional unterschiedlich ab 03/04/05/2008 } \\
\text { 3,3\% Stufenerhöhung ab 04/05/06/2009, Laufzeit bis } \\
\text { 03/04/05/2010 } \\
\text { Einmalzahlung von 0,5\% für die ersten } 13 \text { Monate }\end{array}$ \\
\hline 30.04 .2008 & Deutsche Post AG & $\begin{array}{l}200 € \text { Pauschale insgesamt für Mai-Oktober } \\
\mathbf{4 , 0} \% \text { ab 01.11.08 } \\
\mathbf{3 , 0} \% \text { Stufenerhöhung ab 01.12.09, Laufzeit bis } 30.06 .10\end{array}$ \\
\hline 29.05 .2008 & $\begin{array}{l}\text { Energiewirtschaft } \\
\text { Nordrhein-Westfalen } \\
\text { (GWE) }\end{array}$ & $\begin{array}{l}\text { 4,0 \% ab 01.07.08 } \\
3,5 \% \text { Stufenerhöhung ab 01.07.09, Laufzeit bis 30.06.10 }\end{array}$ \\
\hline 10.07.2008 & $\begin{array}{l}\text { Einzelhandel } \\
\text { Baden-Württemberg }\end{array}$ & $\begin{array}{l}400 € \text { Pauschale insgesamt für April 2007-März } 2008 \\
3,0 \% \text { ab 01.04.08, Laufzeit bis 31.03.09 }\end{array}$ \\
\hline 01.08 .2008 & Deutsche Lufthansa AG & $\begin{array}{l}5,1 \% \text { ab } 01.07 .08 \\
2,3 \% \text { Stufenerhöhung ab 01.07.09 } \\
\text { Einmalzahlung und ergebnisabhängige Komponente von bis } \\
\text { zu 2,4 \% der Jahresgrundvergütung, Laufzeit bis } 28.02 .10\end{array}$ \\
\hline 12.11 .2008 & Metallindustrie & $\begin{array}{l}510 € \text { Pauschale insgesamt für November 2008-Januar } 2009 \\
2,1 \% \text { ab 01.02.09 } \\
2,1 \% \text { ab 01.05.09 (auf Basis des Entgelts Nov. 2008) } \\
122 € \text { Einmalzahlung im September 2009, Laufzeit bis } \\
30.04 .10 \\
0,4 \% \text { Einmalzahlung jeweils für Januar-April } 2010 \\
\text { (Finanzierung Altersteilzeit) }\end{array}$ \\
\hline
\end{tabular}

2,8 \% ebenfalls ab dem 1.1.2009 mit einer Laufzeit bis Ende 2009. Auch hier gingen dem Abschluss umfangreiche Warnstreiks voraus. Am 16.4.2008 erreichte die IG BCE für die chemische Industrie folgenden Abschluss: Regional unterschiedlich gab es ab März/April/Mai 2008 eine Tarifanhebung von $4,4 \%$, gefolgt von einer Stufenerhöhung von 3,3\% ab April/Mai/Juni 2009. Für die ersten 13 Monate wurde eine zusätzliche Einmalzahlung von 0,5 \% gezahlt. Die Gesamtlaufzeit beträgt 25 Monate.

In anderen Branchen gelangen ebenfalls Abschlüsse oberhalb der Inflationsrate: In der Landwirtschaft sieht die Bundesempfehlung eine Erhöhung von 3,8 \% ab dem 1.3.2008 und eine Stufenerhöhung von 3,2 \% ab dem 1.2.2009 bei einer Laufzeit bis Ende März 2010 vor. In der Textil- von $3,9 \%$ ab dem 1.5.2008 sowie eine Stufenerhöhung von 2,9\% ab dem 1.5.2009 für weitere zwölf Monate.

Geringere Tariferhöhungen wurden im Einzelhandel vereinbart: Dort gelang am 10.7.2008 erst nach mehr als einjährigen Verhandlungen der erste regionale Tarifabschluss der Branche in Baden-Württemberg. Er beinhaltete eine Pauschalzahlung von $400 €$ für die ersten zwölf Monate, ab April 2008 erhöhten sich die Tarife für die folgenden zwölf Monate um 3,0\%. Im Brauereigewerbe Nordrhein-Westfalen gab es ebenfalls ein Plus von 3,0 \% für das Jahr 2008. Auch im Hotel- und Gaststättengewerbe Nordrhein-Westfalen betrug die Tariferhöhung 3,0 \% ab 1.3.2008, ein Jahr später folgt einer Stufenerhöhung um 2,5 \% mit einer Laufzeit bis Ende Mai 2010.

Im Kfz-Gewerbe gelang der erste regionale Abschluss in Hessen mit einer Tariferhöhung von 2,5 \% ab April 2008, einer Stufenerhöhung von 2,0 \% ab Dezember 2008 und ein Jahr später weiteren 2,0 \%. Ähnliche Abschlüsse gab es auch in anderen regionalen Tarifbereichen. In NordrheinWestfalen ließ der zuständige Verband die Verhandlungen mit der IG Metall kurz vor der endgültigen Einigung platzen und gab sein tarifpolitisches Mandat ganz auf. Kurz zuvor hatte der Verband mit der konkurrierenden „Christlichen Gewerkschaft Metall“ einen Tarifvertrag zu deutlich schlechteren Konditionen abgeschlossen. Die IG Metall versuchte anschließend, über Firmentarifverträge die Tarifbindung schrittweise wiederherzustellen.

Bei der Deutschen Lufthansa setzte ver.di für das Bodenpersonal nach viertägigem Arbeitskampf Anfang August Gehaltssteigerungen von $5,1 \%$ rückwirkend ab Anfang Juli durch, gefolgt von einer Stufenerhöhung von 2,3 \% ein Jahr später sowie Pauschal- und ergebnisabhängigen Einmalzahlungen.

In der zweiten Jahreshälfte konzentrierte sich das Tarifgeschehen auf die $\mathrm{Me}$ tallindustrie (ausführlich dazu Bispinck/ WSI-Tarifarchiv 2008a). Die IG Metall hatte unter dem Eindruck der lange Zeit hervorragenden Konjunkturdaten und Bilanzzahlen der Metallbranchen eine Tarifforderung von $8 \%$ aufgestellt und konstatierte einen "großen Nachholbedarf in Sachen Gerechtigkeit und Binnenwachstum" (Huber 2008; IG Metall 2008). Nach massiven Warnstreiks im gesamten Bundesgebiet gelang am 12.11. ein Abschluss, der eine Pauschalzahlung von $510 €$ sowie Tarifer- 
höhungen von 2,1 \% ab Februar und weiteren 2,1\% ab Mai 2009 vorsieht. Außerdem gibt es im September 2009 eine Einmalzahlung von $122 €$. Die zweite Stufe kann verschoben und die Einmalzahlung gekürzt werden.

Die Tarifverhandlungen im privaten Bankgewerbe, wo ver.di eine Gehaltssteigerung von $8 \%$, mindestens aber $260 €$ gefordert hatte, wurden angesichts der Bankenkrise im Oktober ausgesetzt. Zu einem Abschluss ist es bis zum Redaktionsschluss dieses Berichts nicht gekommen.

\subsection{WEITERE TARIFPOLITISCHE STATIONEN}

Im März konnte der seit Herbst 2007 laufende Tarifkonflikt zwischen der Deutschen Bahn AG sowie den drei Gewerkschaften Transnet, GDBA und GDL ${ }^{1}$ beigelegt werden (Bispinck/WSI-Tarifarchiv 2008b). Die GDL erhielt die tarifpolitische Zuständigkeit für die Lokführer. Die GDL sowie die Tarifgemeinschaft aus Transnet und GDBA verpflichteten sich, die Tarifverträge der jeweils anderen Gewerkschaft anzuerkennen. Transnet und GDBA vereinbarten ein Tarifwerk zu einem neuen Entgeltsystem, das aus einem Basistarifvertrag und sechs funktionsspezifischen Tarifverträgen besteht.

In der chemischen Industrie vereinbarten die Tarifparteien einen Tarifvertrag „Lebensarbeitszeit und Demografie“. Er fasst Regelungen zu Langzeitkonten, Altersteilzeit, Berufsunfähigkeitszusatzversicherung, Teilrente und zur tariflichen Altersvorsorge zusammen. In der Metallindustrie wurde ein Tarifvertrag zum flexiblen Übergang in die Rente abgeschlossen, der die Altersteilzeit sowie das Altersteilzeitentgelt regelt. Auch in anderen Branchen wurden Tarifregelungen zur Altersteilzeit vereinbart.

\section{Tarifergebnisse in Zahlen und Fakten}

\subsection{LOHN- UND GEHALTSENTWICKLUNG}

Die DGB-Gewerkschaften schlossen 2008 in ganz Deutschland Lohn- und Gehaltstarifverträge für 11,1 Mio. Beschäftigte $\mathrm{ab}$, davon für 9,6 Mio. in den alten und 1,5 Mio.

Tabelle 1: Durchschnittliche Abschlussraten 1) aus der Tarifrunde 2008 - AN in Tarifbereichen ab 1.000 (West) bzw. 500 (Ost) Beschäftigten - in 1.000 -

\begin{tabular}{lcccc}
\hline & $\begin{array}{c}\text { begünstigte } \\
\text { Arbeitnehmer }\end{array}$ & $\begin{array}{c}\text { Erhöhungsrate } \\
\text { in \% }\end{array}$ & $\begin{array}{c}\text { davon: 2008 in } \\
\text { Kraft getretene } \\
\text { Tariferhöhungen } \\
\text { in \% }\end{array}$ & $\begin{array}{c}\text { davon begünstigte } \\
\text { Arbeitnehmer }\end{array}$ \\
\hline Gesamt & $11.102,3$ & 5,2 & 4,0 & $7.585,1$ \\
West & $9.594,5$ & 5,1 & 3,9 & $6.440,1$ \\
Ost & $1.507,8$ & 6,2 & 5,0 & $1.145,0$ \\
\hline
\end{tabular}

1) Einschließlich Stufenerhöhungen, ohne Pauschal- und Einmalzahlungen sowie

leistungsorientierte Vergütungsbestandteile.

Quelle: WSI-Tarifarchiv, Stand: 31.12.2008.

WSI MITTELUNGEN

Tabelle 2: Laufzeit der Tarifverträge - in Monaten -

\begin{tabular}{lcccccccccc}
\hline & $\mathbf{1 9 9 9}$ & $\mathbf{2 0 0 0}$ & $\mathbf{2 0 0 1}$ & $\mathbf{2 0 0 2}$ & $\mathbf{2 0 0 3}$ & $\mathbf{2 0 0 4}$ & $\mathbf{2 0 0 5}$ & $\mathbf{2 0 0 6}$ & $\mathbf{2 0 0 7}$ & $\mathbf{2 0 0 8}$ \\
\hline West & 13,8 & 21,5 & 14,1 & 18,1 & 20,4 & 21,8 & 25,2 & 21,6 & 22,2 & 22,2 \\
Ost & 14,7 & 23,3 & 16,4 & 19,7 & 21,0 & 22,0 & 28,4 & 24,7 & 21,9 & 23,4 \\
\hline \multicolumn{1}{l}{ Quelle: WSI-Tarifarchiv, Stand: 31.12 .2008}$.
\end{tabular}

in den neuen Bundesländern. Das entspricht rund $57 \%$ der von Tarifverträgen erfassten Beschäftigten. Für weitere 4,6 Mio. Beschäftigte traten Stufenerhöhungen in Kraft, die bereits 2007 oder früher vereinbart worden waren. Bei rund 3,7 Mio. Beschäftigten liefen 2008 oder früher die Vergütungstarifverträge aus, aber es kam bis zum Jahresende (noch) nicht zu Neuabschlüssen bzw. es traten keine Tarifanhebungen in Kraft.

\section{ABSCHLUSSRATE}

Die tarifliche Abschlussrate belief sich gesamtwirtschaftlich im Durchschnitt auf 5,2\% (2007: 5,2\%), in Westdeutschland betrug die Rate 5,1\%, in Ostdeutschland 6,2\%. Die Abschlussrate schließt alle, ggf. auch 2009 und später in Kraft tretende tabellenwirksame Erhöhungen ein. Nicht berücksichtigt werden Pauschalzahlungen und zusätzliche Einmalzahlungen, die sich nicht dauerhaft in den Tariftabellen niederschlagen. Die Spannweite der durchschnittlichen Gesamtabschlussraten reicht von $3,0 \%$ im Wirtschaftsbereich Handel, $4,1 \%$ im Nahrungs- und Genussmittelgewerbe, 4,4 \% im Investitionsgütergewerbe über 5,4 \% im Bereich Energie- und Wasserversorgung, Bergbau, 6,8 \% im Grundstoff- und Produktionsgütergewerbe, 7,0 \% im Bereich Gartenbau, Land- und Forstwirtschaft bis zu 8,3\% im Bereich Gebietskörperschaften, Sozialversicherung.

Diese Gesamtabschlussraten sind allerdings von begrenzter Aussagekraft, weil sie sich immer auf die gesamte, je nach Tarifbereich sehr unterschiedlich lange Laufzeit der Tarifabkommen beziehen. Berücksichtigt man lediglich die im Jahr 2008 ab- geschlossenen und auch in Kraft getretenen Tariferhöhungen, ergibt sich eine Abschlussrate von 4,0\% (West: 3,9\%, Ost: 5,0 \%). Differenziert man diese Größe nach Wirtschaftsbereichen, dann ergibt sich für 2008 eine Streuung zwischen 3,0 \% und 5,6 \%. Zu berücksichtigen ist, dass die Erhöhungen zu unterschiedlichen Zeitpunkten im Jahresverlauf wirksam wurden.

Wie bereits in den Vorjahren spielten auch im Jahr 2008 „Nullmonate“ bei den Tarifabschlüssen eine bedeutende Rolle. Für rund 7,9 Mio. (2007: 8,2 Mio.), das entspricht rund $71 \%$ der von Neuabschlüssen begünstigten Beschäftigten, gab es Tarifabschlüsse mit verzögerter Anpassung der Lohn- und Gehaltserhöhungen. 47,3\% mussten zwischen einem und drei Monate auf die reguläre Tariferhöhung warten, für weitere $2,5 \%$ vergingen vier bis fünf Monate bis zur ersten Tarifsteigerung, gut $21 \%$ mussten sogar sechs und mehr Monate ohne Tabellenerhöhung akzeptieren. Als Ausgleich vereinbarten die Gewerkschaften für gut vier Fünftel $(81,5 \%)$ der davon betroffenen Beschäftigten Pauschalzahlungen, die durchschnittlich $116 €$ (West: $118 €$, Ost: $101 €$ ) im Monat betrugen.

\section{LAUFZEITEN}

Der seit einigen Jahren zu beobachtende Trend zu längeren Laufzeiten hat sich im vergangenen Jahr fortgesetzt (Tabelle 2).

\footnotetext{
1 Transnet-Gewerkschaft der Eisenbahner Deutschlands; die heutige Verkehrsgewerkschaft GDBA wurde gegründet als Gewerkschaft Deutscher Bundesbahnbeamten und Anwärter; GDL - Gewerkschaft Deutscher Lokomotivführer.
} 


\begin{tabular}{lccc}
\hline Tabelle 3: Tarifsteigerung 20081) - in \% - & & & \\
\hline Wirtschaftsbereich & Ost & West & Gesamt \\
\hline Gartenbau, Land- und Forstwirtschaft & 3,9 & 3,6 & 3,7 \\
Energie- und Wasserversorgung, Bergbau & 3,6 & 2,8 & 3,0 \\
Grundstoff- und Produktionsgütergewerbe & 3,7 & 3,2 & 3,3 \\
Investitionsgütergewerbe & 2,6 & 2,6 & 2,6 \\
Verbrauchsgütergewerbe & 2,7 & 2,9 & 2,8 \\
Nahrungs- und Genussmittelgewerbe & 2,7 & 2,5 & 2,5 \\
Baugewerbe & 3,0 & 3,0 & 3,0 \\
Handel & 1,7 & 1,9 & 1,9 \\
Verkehr und Nachrichtenübermittlung & 3,9 & 3,4 & 3,5 \\
Kreditinstitute, Versicherungsgewerbe & 2,7 & 2,7 & 2,7 \\
Priv. Dienstleistungen, Organ. o. Erwerbszweck & 3,3 & 3,0 & 3,0 \\
Gebietskörperschaften, Sozialversicherung & 8,7 & 3,2 & 4,4 \\
\hline Gesamte Wirtschaft & $\mathbf{4 , 0}$ & $\mathbf{2 , 7}$ & $\mathbf{2 , 9}$ \\
\hline 1) Jahresbezogene Erhöhung der tariflichen Grundvergütung 2008 gegenüber 2007. & & WS & \\
Quelle: WSI-Tarifarchiv, Stand: 31.12.2008. & & MITTEILUNGEN
\end{tabular}

\begin{tabular}{|c|c|c|c|c|c|c|c|c|c|}
\hline 1999 & 2000 & 2001 & 2002 & 2003 & 2004 & 2005 & 2006 & 2007 & 2008 \\
\hline 91,5 & 91,9 & 92,3 & 92,8 & 93,4 & 94,0 & 94,6 & 95,1 & 95,2 & 96,8 \\
\hline
\end{tabular}

Die Laufzeit der Vergütungstarifverträge im Jahr 2008 beträgt durchschnittlich rund 1,1 Mio. Beschäftigte (10,0 \%) laufen die Abkommen zwischen elf und 17 Monaten, für 3,5 Mio. (31,4 \%) 18 Monate, für 6 Mio. (54,1 \%) 24 Monate und länger, der Rest verteilt sich auf unterschiedliche Laufzeiten. Einen nennenswerten Unterschied zwischen den alten und den neuen Bundesländern gibt es nicht.

\section{JAHRESBEZOGENE TARIFSTEIGERUNG}

Bei der jahresbezogenen Steigerung der tariflichen Grundlöhne und -gehälter werden im Unterschied zur tariflichen Abschlussrate die Auswirkungen aus der (oft unterschiedlichen) Lage und Laufzeit der Tarifabkommen berücksichtigt. Auch werden ggf. im Berichtsjahr wirksam werdende Abschlüsse aus den Vorjahren sowie zusätzliche Einmalzahlungen und Pauschalzahlungen als Ausgleich für Abschlussverzögerungen mit einbezogen. Die jahresbezogene Tarifsteigerung setzt die durchschnittliche tarifliche Grundvergütung des gesamten Jahres 2008 zum Vorjahr in Be22,4 Monate (2007: 22,2 Monate). Für zug und erfasst insgesamt 15,8 Mio. Arbeitnehmerinnen und Arbeitnehmer. Diese kalenderjährliche Steigerung der Tarifverdienste 2008 gegenüber 2007 betrug für ganz Deutschland 2,9\% (Tabelle 3). Am höchsten fiel die jahresbezogene Tarifsteigerung mit 4,4 \% im Bereich Gebietskörperschaften, Sozialversicherung aus, gefolgt vom Bereich Gartenbau, Land- und Forstwirtschaft mit 3,7 \% und dem Bereich Verkehr und Nachrichtenübermittlung mit $3,5 \%$. Unterdurchschnittlich war die Tarifsteigerung im Bereich Kreditinstitute, Versicherungsgewerbe mit 2,7 \%, im Investitionsgütergewerbe mit 2,6\% sowie im Nahrungs- und Genussmittelgewerbe mit $2,5 \%$. Am niedrigsten fiel die Steigerung im Handel mit $1,9 \%$ aus.

In Ostdeutschland lag die kalenderjährliche Erhöhung mit 4,0\% deutlich höher als in Westdeutschland mit 2,7 \%. Dies hängt maßgeblich mit der Anpassung des Tarifniveaus Ost im öffentlichen Dienst zusammen.

In Tarifbereichen mit 1,1 Mio. Beschäftigten liefen Vergütungstarifverträge im Jahr 2008 aus, ohne dass bis zum Jahresende neue Abschlüsse getätigt wurden. Be-

\begin{tabular}{|c|c|c|c|c|c|c|c|c|c|c|}
\hline \multicolumn{11}{|c|}{$\begin{array}{l}\text { Tabelle 5: Tarifliche Ausschöpfung des neutralen Verteilungs- } \\
\text { spielraums }{ }^{1)}-\text { in } \% \text { - }\end{array}$} \\
\hline 1998 & 1999 & 2000 & 2001 & 2002 & 2003 & 2004 & 2005 & 2006 & 2007 & 2008 \\
\hline$-0,3$ & 1,0 & $-1,6$ & $-1,7$ & $-0,2$ & 0,2 & $-0,2$ & $-1,8$ & $-2,7$ & $-0,6$ & 0,4 \\
\hline \multirow{2}{*}{\multicolumn{11}{|c|}{$\begin{array}{l}\text { 1) Saldo aus Verteilungsspielraum (Anstieg von Verbraucherpreisen + Arbeitsproduktivität/ } \\
\text { Stunde) und Tariferhöhung. }\end{array}$}} \\
\hline & & & & & & & & & & WSI MITTEILUNGEN \\
\hline
\end{tabular}

zieht man diese Tarifbereiche mit ein, dann sinkt die jahresbezogene Tarifsteigerung geringfügig von 2,9 auf 2,8 \%. In Tarifbereichen mit weiteren 2,5 Mio. Beschäftigten sind die Tarifverträge bereits 2007 oder in den Jahren zuvor ausgelaufen ohne nachfolgende Abschlüsse. Setzt man die Tarifsteigerung für diese Bereiche mit $0 \%$ an, dann sinkt die jahresbezogene Tarifsteigerung für 2008 insgesamt auf gut 2,4\%.

Der Stand der tariflichen Lohnangleichung an das Westniveau kann an der Entwicklung der tariflichen Grundvergütung festgemacht werden. Für den Stichtag 31.12.2008 ergibt sich dabei folgendes Bild (Tabelle 4): Auf Basis von rund 50 Tarifbereichen/-branchen mit 1,8 Mio. erfassten Beschäftigten errechnet sich ein durchschnittliches Tarifniveau von 96,8\%. Damit ergibt sich gesamtwirtschaftlich ein Anstieg gegenüber dem Vorjahr um 1,6 Prozentpunkte. Entscheidend hierfür war die Anhebung des Tarifniveaus Ost im öffentlichen Dienst von 92,5 bzw. $97 \%$ auf $100 \%$ der Westtarife.

Die Steigerung der Ausbildungsvergütungen ist im vergangenen Jahr niedriger ausgefallen als 2007. Nach Berechnungen des Bundesinstituts für Berufsbildung ergibt sich ein Anstieg von 2,2 \% (West: $2,0 \%$, Ost: $2,9 \%$ ), der damit unter der Steigerung der tariflichen Grundvergütungen und auch unterhalb des Anstiegs der Verbraucherpreise liegt (BIBB 2008). Je nach Tarifbereich verbergen sich hinter diesen Durchschnittszahlen große Unterschiede: Gemessen an der Ausbildungsvergütung im dritten Ausbildungsjahr lagen die Beträge Ende 2008 in Vierfünftel der für diesen Tarifbericht ausgewählten 26 Tarifbereiche (West/Ost) unverändert auf dem Vorjahresniveau. Im Übrigen variierten die Anhebungen zwischen 1,1\% im Versicherungsgewerbe und 10,3\% in der Stahlindustrie.

\section{TARIF-, EFFEKTIV- UND REALLOHNENTWICKLUNG}

Die durchschnittliche jahresbezogene Tarifsteigerung 2008 von 2,9 \% liegt gerade einmal 0,3 Prozentpunkte über dem Anstieg der Lebenshaltungskosten von 2,6\%. Real stiegen die tariflichen Vergütungen im gesamtwirtschaftlichen Durchschnitt also nur geringfügig. Angesichts des leicht rückläufigen Produktivitätsanstiegs wurde der kostenneutrale Verteilungsspielraum aus Preissteigerung $(+2,6 \%)$ und Produkti- 
vitätszuwachs $(-0,1 \%)$ mehr als ausgeschöpft. Über einen längeren Zeitraum betrachtet, ergibt sich folgendes Bild:

Betrachtet man die Effektiveinkommensentwicklung in Gesamtdeutschland, so entsteht ein ungünstigerer Eindruck (vgl. Statistisches Bundesamt 2009): Die Summe der Bruttolöhne und -gehälter stieg 2008 um $3,6 \%$. Je Beschäftigten und je Arbeitnehmerstunde ergibt sich ein Anstieg um 2,3 \%. Daraus ergibt sich, dass die Bruttoreallöhne 2008 zum fünften Mal in Folge gesunken sind. Die Differenz zwischen Tarif- und Effektivlohnentwicklung belegt die Fortsetzung der negativen Lohndrift.

Erstmals seit über zehn Jahren sind die Lohnstückkosten (Arbeitskosten/Produktivität) im vergangenen Jahr mit 2,1 \% etwas stärker angestiegen. Im europäischen Vergleich blieb es bei dem bekannten Bild: In allen wichtigen Konkurrenzländern innerhalb der EU wuchsen die Lohnstückkosten erheblich stärker als in Deutschland (IMK 2008).

\subsection{ARBEITSZEIT}

Die gesamtwirtschaftlichen Eckdaten der tariflichen Wochenarbeitszeit sind im Laufe des vergangenen Jahres weitgehend gleich geblieben. Die tarifliche Wochenarbeitszeit betrug Ende 2008 im gesamtwirtschaftlichen Durchschnitt in ganz Deutschland 37,6 Stunden (West: 37,4 und Ost: 38,8 Stunden) (Übersicht 3). Angesichts der zahlreichen arbeitszeitbezogenen Öffnungsklauseln und Flexi-Bestimmungen (u.a. mit Kontenregelungen und langen Ausgleichszeiträumen) müssen die gesamtwirtschaftlichen und branchenbezogenen Zahlen zur tariflichen Arbeitszeitdauer eher als Referenzgrößen denn als Beschreibung der tatsächlichen Arbeitszeitstandards angesehen werden.

Die tarifliche Urlaubsdauer (Endstufe) beträgt im gesamtdeutschen Durchschnitt unverändert 30,0 Tage (West: 30,1 und Ost: 29,5 Tage). Errechnet man auf Basis dieser und weiterer Einzelkomponenten die tarifliche Jahresarbeitszeit, so ergibt sich ein gesamtdeutscher Durchschnitt von 1.656,2 Stunden, für Westdeutschland $1.644,8$ und für Ostdeutschland $1.715,4$ Stunden.

\subsection{ARBEITSKAMPFGESCHEHEN}

Die Tarifauseinandersetzungen waren auch im Jahr 2008 häufig von Arbeitskampf-

Tabelle 6: Wirtschafts- und Verteilungsdaten 2007-2008

- Veränderung gegenüber dem Vorjahr in \% -

\begin{tabular}{lcc} 
& $\mathbf{2 0 0 7}$ & $\mathbf{2 0 0 8}$ \\
\hline Bruttoinlandsprodukt & 2,5 & 1,3 \\
Verbraucherpreise & 2,2 & 2,6 \\
Produktivität/Stunde & 0,8 & $-0,1$ \\
Tarifentgelte/Stunde $1^{-}$ & 2,2 & 2,9 \\
Bruttoverdienste/Stunde & 1,4 & 2,3 \\
Lohnstückkosten/Stunde & 0,0 & 2,1 \\
\hline 1) Je tarifliche Arbeitsstunde. & WSI MITTEILUNGEN \\
Quelle: Stat. Bundesamt; WSI-Tarifarchiv. &
\end{tabular}

\begin{tabular}{|c|c|c|c|}
\hline \multicolumn{4}{|c|}{ Übersicht 3: Tarifliche Arbeitszeitregelungen 2008} \\
\hline Tarifregelung & Ost & West & Gesamt \\
\hline Wochenarbeitszeit (Std.) & 38,8 & 37,4 & 37,6 \\
\hline \multicolumn{4}{|l|}{ Anteil der Beschäftigten (in \%) mit: } \\
\hline bis zu 35 & 5,9 & 25,5 & 22,3 \\
\hline $36-37$ & 5,6 & 9,8 & 9,2 \\
\hline $37,5-38,5$ & 31,0 & 36,0 & 35,1 \\
\hline 39-40 und mehr & 57,2 & 28,2 & 32,7 \\
\hline Urlaub (Arbeitstage) ${ }^{1}$ & 29,5 & 30,1 & 30,0 \\
\hline Jahresarbeitszeit (Std.) & $1.715,4$ & $1.644,8$ & $1.656,2$ \\
\hline $\begin{array}{l}\text { 1) Endstufe. } \\
\text { Quelle: WSI-Tarifarchiv, Stand: } 31.12 .2008 .\end{array}$ & \multicolumn{3}{|c|}{ WSI MITTEILUNGEN } \\
\hline
\end{tabular}

Tabelle 7: Tarifliche Wochen- und Jahresarbeitszeit 1999-2008 - in Std. -

\begin{tabular}{rrrrrrrrrrrr}
\hline & & 1999 & $\mathbf{2 0 0 0}$ & $\mathbf{2 0 0 1}$ & $\mathbf{2 0 0 2}$ & $\mathbf{2 0 0 3}$ & $\mathbf{2 0 0 4}$ & $\mathbf{2 0 0 5}$ & $\mathbf{2 0 0 6}$ & $\mathbf{2 0 0 7}$ & $\mathbf{2 0 0 8}$ \\
\hline Woche & $\mathrm{G}$ & 37,7 & 37,7 & 37,7 & 37,7 & 37,7 & 37,6 & 37,6 & 37,6 & 37,6 & 37,6 \\
& $\mathrm{~W}$ & 37,4 & 37,4 & 37,4 & 37,4 & 37,4 & 37,4 & 37,4 & 37,4 & 37,4 & 37,4 \\
& $\mathrm{O}$ & 39,2 & 39,1 & 39,1 & 39,1 & 39,0 & 38,9 & 39,0 & 38,9 & 38,8 & 38,8 \\
\hline Jahr & $\mathrm{G}$ & 1658,6 & 1657,8 & 1656,3 & 1656,0 & 1656,3 & 1655,7 & 1655,6 & 1657,0 & 1656,8 & 1656,2 \\
& W & 1642,8 & 1642,5 & 1641,9 & 1642,6 & 1643,5 & 1643,3 & 1643,2 & 1644,7 & 1645,2 & 1644,8 \\
& O & 1729,9 & 1727,7 & 1724,2 & 1722,7 & 1721,9 & 1719,2 & 1718,9 & 1720,3 & 1716,4 & 1715,4 \\
\hline \multicolumn{7}{l}{ Stand: jeweils in Kraft zum 31.12. jeden Jahres. G=Gesamt, W=West, O=Ost. } & & WSI MITTEILUNGEN \\
Quelle: WSI-Tarifarchiv, Stand: 31.12.2008.
\end{tabular}

maßnahmen begleitet. Dabei zeigt ein genauerer Blick, dass Anlass, Umfang und Intensität erheblich differieren. $\mathrm{Zu}$ unterscheiden sind in formaler Hinsicht verhandlungsbegleitende Warnstreiks und reguläre Arbeitsniederlegungen nach vorheriger Urabstimmung. Von der strategischen Anlage her geht es entweder um offensive Erzwingungsstreiks zur Durchsetzung gewerkschaftlicher Forderungen oder um Abwehrstreiks gegen die Verschlechterung von Tarifstandards (Dribbusch 2008). Inhaltlich standen im Tarifjahr 2008 Lohnforderungen im Vordergrund, aber Konfliktgegenstände waren auch manteltarifliche Fragen der Arbeitszeit, des Rentenübergangs und ganz generell die Aufrechterhaltung der Tarifbindung.

\section{WARNSTREIKS}

In einigen Branchen ist inzwischen fast jede Tarifrunde von Warnstreiks begleitet.
Dies gilt z. B. für die Metall- und Elektroindustrie, in der in den vergangenen elf Jahren lediglich in zwei Jahren eine Einigung ohne Warnstreiks erreicht werden konnte. In diesem Jahr beteiligten sich zunächst im Juni 360.000 Metallerinnen und Metaller an Warnstreiks für eine neue tarifliche Altersteilzeitregelung. In der Entgeltrunde im Herbst konnte die IG Metall in mehreren Warnstreikwellen sogar mehr als 600.000 Beschäftigte mobilisieren. Zwei massive Warnstreikwellen mit $430.000 \mathrm{Be}$ teiligten reichten letztlich auch im öffentlichen Dienst (Bund, Gemeinden), um nach langwierigen Verhandlungen sowie einer gescheiterten Schlichtung doch noch eine Einigung zu erzielen. Weitere Warnstreiks gab es in dieser Tarifrunde u.a. auch in der Stahlindustrie, in der Papierverarbeitung, in der Textil- und Bekleidungsindustrie, in der Entsorgungswirtschaft, in der Energiewirtschaft und bei der Deutschen Post. 


\begin{tabular}{|c|c|c|c|}
\hline \multicolumn{4}{|c|}{$\begin{array}{l}\text { Übersicht 4: (Warn-)Streiks im Jahr } 2008 \text { in ausgewählten } \\
\text { Tarifbereichen }\end{array}$} \\
\hline Branche & Art/Dauer & Gegenstand & Beteiligte \\
\hline Bankgewerbe & $\begin{array}{l}\text { Protestaktionen/ } \\
\text { Warnstreiks 3.7.-30.9 }\end{array}$ & Gehalt & 19.800 \\
\hline Deutsche Lufthansa AG & $\begin{array}{l}\text { Streik } \\
28 .-31.7 .\end{array}$ & Lohn, Gehalt & k. A. \\
\hline Einzelhandel & (Warn-)Streiks & Lohn, Gehalt, Mantel & $180.000^{1)}$ \\
\hline Kfz-Gewerbe NRW & $\begin{array}{l}\text { Warnstreiks } \\
\text { 11.-14.3. }\end{array}$ & Lohn, Arbeitszeit & 8.100 \\
\hline Metallindustrie & $\begin{array}{l}\text { Warnstreiks } \\
\text { 3.-26.06 }\end{array}$ & Altersteilzeit & 360.000 \\
\hline Metallindustrie & $\begin{array}{l}\text { Warnstreiks } \\
\text { 1.-10.11. }\end{array}$ & Lohn, Gehalt & 611.500 \\
\hline $\begin{array}{l}\text { Öffentlicher Dienst } \\
\text { (Bund, Gemeinden) }\end{array}$ & $\begin{array}{l}\text { Warnstreiks } \\
\text { 14.2.-6.3. }\end{array}$ & Entgelt & 430.000 \\
\hline Papierverarbeitung & $\begin{array}{l}\text { Warnstreiks } \\
\text { 10.4.-8.5. }\end{array}$ & Lohn, Gehalt & 6.000 \\
\hline Stahlindustrie NRW und Ost & $\begin{array}{l}\text { Warnstreiks } \\
\text { 1.-19.2. }\end{array}$ & Lohn, Gehalt & 44.000 \\
\hline Textil-/Bekleidung West & $\begin{array}{l}\text { Warnstreiks } \\
1 .-10.3 .\end{array}$ & Lohn, Gehalt & 18.100 \\
\hline
\end{tabular}

\section{ERZWINGUNGSSTREIKS}

$\mathrm{Zu}$ einem klassischen Erzwingungsstreik rief ver.di nach erfolgreicher Urabstimmung in der diesjährigen Tarifrunde bei der Deutschen Lufthansa auf. Nach vier, von einem großen Medienecho begleiteten Streiktagen kam es hier zur Einigung.

Die längsten von Streiks begleiteten Tarifrunden fanden im Einzelhandel sowie im öffentlichen Dienst Berlins statt. In der über ein Jahr dauernden Einzelhandelstarifrunde führte ver.di zwischen Mai 2007 und Juli 2008 im gesamten Bundesgebiet mehr als 6.400 Streikaktionen durch. Diese reichten von Tagesstreiks bis zu einwöchigen Arbeitsniederlegungen. Die Unternehmerseite reagierte in großem Stil mit organisiertem Streikbruch. Im öffentlichen Dienst in Berlin waren seit August 2007 immer wieder Arbeitskampfmaßnahmen notwendig, bis schließlich im November 2008 eine Einigung erzielt werden konnte. In beiden Fällen war die Länge der Auseinandersetzung Ausdruck einer Pattsituation, in der es der Gewerkschaftsseite Probleme bereitete, die Blockade der Arbeitgeberseite durch den Aufbau effektiven wirtschaftlichen bzw. politischen Drucks zu überwinden.

\section{ABWEHRSTREIKS}

In mehreren Fällen mussten sich Gewerkschaften mit Tarifflucht auseinandersetzen. Hierbei erzwang u.a. die IG Metall im KfzGewerbe in Nordrhein-Westfalen durch Streik das Verbleiben der Lueg AG im Metalltarif. Nach einer Woche Streik wurde bei Hanau die Rückkehr in die Tarifbindung erreicht und beim norddeutschen Gerätehersteller Möller-Wedel konnte die IG Metall nach 15-tägigem Streik ebenfalls einen neuen Tarifvertrag durchsetzen.

\section{STREIKS VON BERUFSGEWERK- SCHAFTEN}

Nicht nur die DGB-Gewerkschaften, sondern auch zwei Berufsgewerkschaften führten im Jahr 2008 Arbeitskampfmaßnahmen durch. Dazu gehörte der Marburger Bund der Krankenhausärzte, der in der Tarifrunde an den Unikliniken Warnstreiks einsetzte. Bei der Lufthansa griff nach ver.di auch die Pilotenvereinigung Cockpit auf (Warn-)Streiks vor allem bei einigen Tochtergesellschaften zurück. Die Unabhängige Flugbegleiterorganisation (Ufo) kündigte ebenfalls Kampfaktionen an, sodass nach dem Tarifkonflikt von drei Gewerkschaften (Transnet, GDBA und GDL) mit der Deutschen Bahn erneut eine Debatte über konkurrierende Gewerkschaften und eine mögliche Begrenzung des Streikrechts aufbrach.

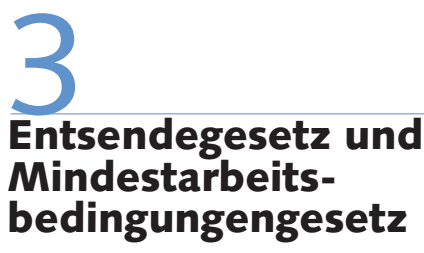

Die Große Koalition hatte sich im Sommer 2007 auf eine Ausweitung des Arbeitneh- der Firma Vacuumschmelze im hessischen merentsendegesetzes sowie auf eine Modernisierung des Mindestarbeitsbedingungengesetzes geeinigt. Anfang Januar 2008 legte Bundesarbeitsminister Scholz die Referentenentwürfe zur Novellierung des Arbeitnehmerentsendegesetzes und des Mindestarbeitsbedingungengesetzes vor. Bis Ende März 2008 konnten die interessierten Tarifvertragsparteien Antrag auf Aufnahme in das Entsendegesetz stellen. Dies taten sie in folgenden Branchen (Bispinck/Schulten 2008):

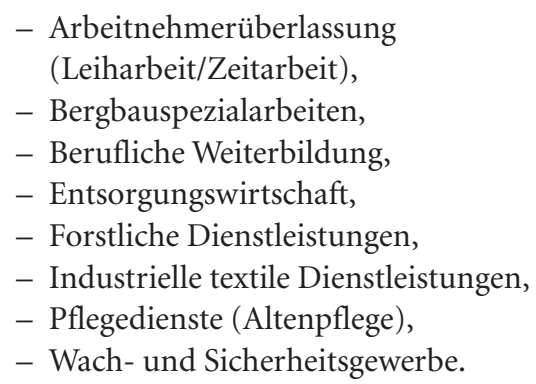

Allerdings lag nicht für alle Branchen bereits ein ausgehandelter Mindestlohntarifvertrag vor. Im Wach- und Sicherheitsgewerbe hatten sich Arbeitgeber und ver.di nicht auf einen Vertrag einigen können. Der Arbeitsgeberverband schloss daraufhin im Juni 2008 mit der christlichen Gewerkschaft Öffentlicher Dienst und Dienstleistungen (GÖD) einen Mindestlohntarifvertrag ab.

Die Gesetzentwürfe des Bundesarbeitsministers blieben in der Koalition umstritten. Erst im Juli einigte sich das Kabinett auf die endgültige Fassung (Bundesratsdrucksache 541/08 und 542/08). In der weiteren politischen Beratung traten erneut Meinungsverschiedenheiten auf. Der Bundesrat formulierte eine Reihe von Bedenken, in der Anhörung des Bundestagsausschusses für Arbeit und Soziales gingen die Meinungen der geladenen Experten weit auseinander (Ausschussdrucksache 16(11)1117 vom 31.10.2008). Von gewerkschaftlicher Seite wurde beim Mindestarbeitsbedingungengesetz vor allem der hohe bürokratische Aufwand bei gleichzeitig geringer Wirksamkeit des Verfahrens kritisiert (DGB 2008). Im Kern bleiben auch bei Umsetzung beider Gesetzesvorhaben gravierende Regelungslücken bestehen, die letztlich nur mit einem allgemeinen, einheitlichen gesetzlichen Mindestlohn geschlossen werden können (Bispinck/Schulten 2008).

Die Koalitionsparteien konnten über Monate hinweg trotz intensiver Beratungen keine Einigung darüber erzielen, wel- 
che Branchen konkret ins Entsendegesetz aufgenommen werden sollten. Während CDU/CSU die Zahl möglichst gering halten wollte und lediglich beim Wach- und Sicherheitsgewerbe Handlungsbedarf sah, wollte die SPD im Grundsatz alle acht Branchen, vor allem aber die Leiharbeitsbranche, in das Gesetz aufnehmen. Erst im Januar 2009 wurde ein Kompromiss gefunden. Sechs Branchen sollen in den Schutzbereich des Arbeitnehmerentsendegesetzes aufgenommen werden. Dazu gehören die Entsorgungsbranche, das Wach- und Sicherheitsgewerbe, die Bergbauspezialdienste, die industriellen Großwäschereien, die Pflegedienste und die berufliche Weiterbildung. Nicht aufgenommen werden sollen die Leiharbeitsbranche und die forstlichen Dienstleistungen. Für die Leiharbeitnehmer soll eine Lohnuntergrenze im Arbeitnehmerüberlassungsgesetz festgehalten werden, die sich an den untersten bestehenden Tarifvergütungen orientieren soll. Die konkrete Umsetzung blieb zwischen den Koalitionsparteien strittig. Sollte der Tarifvertrag der Tarifgemeinschaft Christlicher Gewerkschaften für Zeitarbeit (CGZP) mit dem Arbeitgeberverband AMP zugrunde gelegt werden, beträgt die unterste Tarifgruppe im Westen lediglich 7,21 €, die allerdings in den ersten sechs Beschäftigungsmonaten nochmals um 9,5\% auf $6,53 €$ abgesenkt werden darf. In dem von der DGB-Tarifgemeinschaft Zeitarbeit bereits 2006 abgeschlossenen Mindestlohntarifvertrag mit den beiden größten Arbeitgeberverbänden BZA und iGZ sind dagegen 7,31 € vorgesehen. Die Regelung stieß deshalb auch auf heftige Kritik der DGB-Gewerkschaften („Mogelpackung für Leiharbeit").

Der vom Bundesarbeitsminister auf Basis des Entsendegesetzes für den Bereich der Briefdienstleistungen erlassene Mindestlohn, der zum 1.1.2008 in Kraft trat, wurde im Dezember 2008 vom Oberverwaltungsgericht Berlin-Brandenburg in zweiter Instanz mit der Begründung für rechtswidrig erklärt, dass der Bund seine gesetzliche Ermächtigung überschritten habe und einen Mindestlohn-Tarifvertrag nur auf tariflich nicht gebundene Arbeitnehmer und Arbeitgeber erstrecken dürfe (Oberverwaltungsgericht Berlin-Brandenburg 2008). Die Rechtsverordnung verdränge jedoch bestehende Tarifvereinbarungen. Gemeint waren damit die Tarifverträge, die der Arbeitgeberverband Neue Brief- und Zustelldienste sowie kurz darauf

\begin{tabular}{|c|c|c|c|}
\hline \multicolumn{4}{|c|}{ Übersicht 5: Neue tarifliche Mindestlöhne nach dem AEntG } \\
\hline Branche & Beschäftigte & \multicolumn{2}{|c|}{ Mindestlohn } \\
\hline \multicolumn{2}{|c|}{ Aufnahme in das AEntG vom Bundestag beschlossen } & & \\
\hline \multirow{3}{*}{$\begin{array}{l}\text { Bergbauspezial- } \\
\text { arbeiten }\end{array}$} & 2.500 & $a b 01 / 2009$ & ab 07/2009 \\
\hline & Mindestlohn I & 10,96 & 11,17 \\
\hline & $\begin{array}{l}\text { Mindestlohn II } \\
\text { (Hauer/Facharbeiter) }\end{array}$ & 12,17 & 12,41 \\
\hline Berufliche Weiterbildung & 23.000 & - & - \\
\hline West & Verwaltungsangestellte/r & 10,71 & - \\
\hline Ost & & 9,53 & - \\
\hline West & $\begin{array}{l}\text { Pädagogische/r } \\
\text { Mitarbeiter/in }\end{array}$ & 12,28 & - \\
\hline Ost & & 10,93 & - \\
\hline \multirow{2}{*}{$\begin{array}{l}\text { Entsorgungs- } \\
\text { wirtschaft }\end{array}$} & 130.000 & ab 05/2009 & - \\
\hline & & 8,02 & - \\
\hline $\begin{array}{l}\text { Industrielle } \\
\text { textile Dienste }\end{array}$ & 35.000 & ab 03/2009 & - \\
\hline West & Mindestentgelt & $1.480,741)$ & - \\
\hline Ost & Mindestentgelt & $1.393,741)$ & - \\
\hline $\begin{array}{l}\text { Pflegedienste } \\
\text { (Altenpflege) }\end{array}$ & 565.000 & - & - \\
\hline \multirow[t]{2}{*}{ Wach- und Sicherheitsgew. ${ }^{3}$} & 177.000 & $\mathrm{ab} 05 / 2009$ & - \\
\hline & & $6,00-8,32$ & - \\
\hline \multicolumn{4}{|c|}{ Nicht zur Aufnahme in das AEntG vorgesehen: } \\
\hline \multirow{2}{*}{$\begin{array}{l}\text { Private Forstdienst- } \\
\text { leister }\end{array}$} & 10.000 & $01 / 2009$ & 07/2009 \\
\hline & Mindestlohn & 9,38 & 10,26 \\
\hline Zeitarbeit ${ }^{4}$ & 630.000 & $a b 01 / 2008$ & - \\
\hline West & Mindestentgelt & 7,31 & - \\
\hline Ost inkl. Berlin & Mindestentgelt & 6,36 & - \\
\hline \multicolumn{4}{|c|}{ 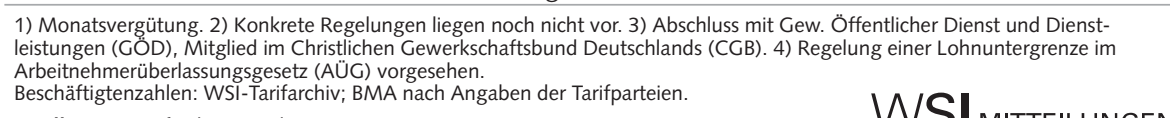 } \\
\hline Quelle: WSI-Tarifarchiv, Stand 30.0 & & & ITTEILUNGEN \\
\hline
\end{tabular}

\begin{tabular}{|c|c|c|}
\hline \multicolumn{3}{|c|}{$\begin{array}{l}\text { Übersicht 6: Tariferhöhungen in } \mathbf{2 0 0 9} \text { - Erhöhungen aus Abschlüssen } \\
\text { 20081) - }\end{array}$} \\
\hline Branche & $\%$ & ab ... 2009 \\
\hline Chemische Industrie West & 3,3 & April-Juni2) \\
\hline Deutsche Post AG & 3,0 & Dezember \\
\hline Energiewirtschaft NRW (GWE) & 3,5 & Juli \\
\hline Hotels u. Gaststätten NRW & 2,5 & März \\
\hline Kfz-Gewerbe Hessen & 2,0 & Dezember \\
\hline Landwirtschaft & 3,3 & Februar \\
\hline \multirow[t]{2}{*}{ Metallindustrie } & 2,1 & Februar \\
\hline & 2,1 & Mai \\
\hline Steinkohlenbergbau & 2,0 & Juli \\
\hline Öffentlicher Dienst Bund, Gemeinden & 2,8 & Januar \\
\hline $\begin{array}{l}\text { 1) Ohne Pauschal- und Einmalzahlungen. 2) Regional unterschiedlich. } \\
\text { Quelle: WSI-Tarifarchiv, Stand: } 31.12 .2008 .\end{array}$ & & TTEILUNGE \\
\hline
\end{tabular}

der Bundesverband der Kurier-, Expressund Postdienste (BdKEP) mit der neu gegründeten Gewerkschaft der Neuen Briefund Zustelldienste (GNBZ) abgeschlossen hatten. Die GNBZ stellt jedoch nach einem Urteil des Kölner Arbeitsgerichts keine Gewerkschaft dar, weil sie aufgrund von finanziellen Zuwendungen seitens der Arbeitgeber nicht die für Gewerkschaften notwendige Unabhängigkeit aufweise. Damit sind die geschlossenen Tarifverträge nichtig. Der Bundesarbeitsminister legte Revision gegen das Urteil des Oberverwaltungsgerichts ein, die Mindestlohnverordnung bleibt bis zur endgültigen Erklärung in Kraft.

Der Handlungsdruck in Sachen Regulierung des Niedriglohnsektors erhöhte sich nach dem Urteil des Europäischen Gerichtshofes vom April 2008 zur Rechtswidrigkeit des niedersächsischen Tariftreuegesetzes. Tariftreueregelungen sind danach 
mit der europäischen Dienstleistungsfreiheit nicht vereinbar (EuGH 2008). Dieses Urteil macht nicht nur einen allgemeinen Mindestlohn, sondern auch eine Reform, die die Allgemeinverbindlichkeit von Tarifverträgen deutlich erleichtert, immer dringlicher (Schulten/Pawicki 2008).

Gültige Mindestlöhne nach dem Entsendegesetz bestanden zum Redaktionsschluss dieses Berichts (Ende Januar 2009) in folgenden Branchen: Abbruch- und Abwrackgewerbe, Bauhauptgewerbe, Briefdienstleistungen, Dachdeckerhandwerk, Elektrohandwerk (Montage), Gebäudereinigerhandwerk, Maler- und Lackiererhandwerk.

\section{4 \\ Ausblick auf die Tarifentwicklung 2009}

In einigen Tarifbereichen wurden bereits Tariferhöhungen für das Jahr 2009 vereinbart, wie Übersicht 6 zeigt. Die im kommenden Jahr in Kraft tretenden Steigerungsraten bewegen sich zwischen 2,0 und $4,2 \%$.

Im Jahr 2009 stehen im Übrigen in zahlreichen Wirtschaftszweigen Tarifverhandlungen an. Ende Dezember 2008 liefen im öffentlichen Dienst die Tarifverträge der Länder aus. Auch die Tarifverträge bei der Deutschen Telekom und für die Zeitarbeit stehen zur Neuverhandlung an. Ende Januar folgte die Deutsche Bahn AG. Ende Februar 2009 endeten u. a. die Verträge in der westdeutschen Textil- und Bekleidungsindustrie. Ende März folgen das Bauhauptgewerbe, die Eisen- und Stahlindustrie sowie Teile des Einzel- und Großhandels, Ende April die Holz und Kunststoff verarbeitende Industrie sowie weitere Teile des Handels. Die Tarifforderungen sind weit gefächert: Im öffentlichen Dienst fordert ver.di $8 \%$ und mindestens $200 €$, bei der Deutschen Bahn AG will Transnet 10 \% höhere Entgelte durchsetzen; in anderen Branchen fordern die Gewerkschaften zwischen 5 und $7 \%$.

\section{LITERATUR}

Bundesinstitut für Berufsbildung (BiBB) (2008): Tarifliche Ausbildungsvergütungen 2008 - Weiterhin nur sehr geringer Anstieg, Pressemitteilung 1 vom 8.1 .

Bispinck, R./WSI-Tarifarchiv (2008a): Tarifpolitischer Jahresbericht 2008: Tarifpolitik in der Finanzmarktkrise, in: WSI-Informationen zur Tarifpolitik, Januar

Bispinck, R./WSI-Tarifarchiv (2008b): Tarifpolitischer Jahresbericht 2007: Gespaltene Tarifentwicklung und verschärfte Gewerkschaftskonkurrenz, in: WSI-Mitteilungen 2, S. 77-84

Bispinck, R. (Hrsg.) (2008): Verteilungskämpfe und Modernisierung. Aktuelle Entwicklungen in der Tarifpolitik, Hamburg

Bispinck, R./Schulten Th. (2008): Aktuelle Mindestlohndebatte: Branchenlösungen oder gesetzlicher Mindestlohn?, in: WSI-Mitteilungen 3, S. $151-158$

Deutscher Gewerkschaftsbund (DGB) (2008): Stellungnahme zum Gesetzentwurf der Bundesregierung eines Ersten Gesetzes zur Änderung des Gesetzes über die Festsetzung von Mindestarbeitsbedingungen sowie zum Entwurf eines Arbeitnehmer-Entsendegesetzes vom 28.10.

Dribbusch, H. (2008): Streiks in Deutschland - Rahmenbedingungen und Entwicklung ab 1990, in: WSI (Hrsg.): WSI-Tarifhandbuch 2008, Frankfurt/Main, S. 55-85

Europäischer Gerichtshof (EuGH) (2008): Urteil des Gerichtshofes in der Rechtssache C-346/06 (Tariftreueregelung in Niedersachsen), 3. April
Huber, B. (2008): Statement anlässlich der Pressekonferenz zur Forderungsempfehlung der Metall- und Elektroindustrie für die Tarifrunde 2008, Frankfurt, 8. 9.

Industriegewerkschaft Metall (IGM) (2008): Tarifrunde 2008/09 in der Metallindustrie. Sozialökonomische Rahmenbedingungen, Wirtschaftspolitische Informationen 06 vom 8.9.

Institut für Makroökonomie und Konjunkturforschung (IMK) (2008): Deutsche Arbeitskosten steigen im europäischen Vergleich nur gering. Auswertung der aktuellen Eurostat-Statistik, IMK-Report 34, November Jänicke, S./Rohnert, R./Wagner, H. (2008): Schlechte Zeiten für mehr Gerechtigkeit?!, in: Sozialismus 12, S. 25-30

Kannegießer, M. (2008): „Im Abschwung höchste Forderung seit 16 Jahren zu stellen, ist leichtfertig " - Gesamtmetall-Informationen für die Presse 25 vom 8.9 .

Oberverwaltungsgericht Berlin-Brandenburg (2008): Postmindestlohnverordnung auch in zweiter Instanz beanstandet, Pressemitteilung 30 vom 18.12 .

Schulten, Th./Pawicki, M. (2008): Tariftreueregelungen in Deutschland Ein aktueller Überblick, in: WSI-Mitteilungen 4, S. 184-190 Statistisches Bundesamt (2009): Volkswirtschaftliche Gesamtrechnung 2008, vorläufige Ergebnisse, Wiesbaden 